\title{
An Improved Scheme to Fingerprint Classification
}

\author{
Weimin Huang and Jian-Kang Wu \\ Kent Ridge Digital Labs *, \\ 21 Heng Mui Keng Terrace, Kent Ridge, \\ Singapore 119613 \\ Email: \{wmhuang, jiankang\}@krdl.org.sg
}

\begin{abstract}
An improved scheme to fingerprint classification is presented in this paper. It is well known that automatic fingerprint identification system usually needs to access very huge data - thousands of fingerprints stored in database. Thus a good automatic classification algorithm is an important module in such a system. Further more, for identification of fingerprint, it also asks few error occurred in its pre-classification stage. The scheme presented here extracts the singularities of fingerprints. Based on those points, the flow-lines which describe the global pattern of fingerprint are obtained with flow-line tracing algorithm using the direction and skeleton information. The properties of the flowlines are analyzed with respect to fingerprint pattern. Then the scheme performs the overlapped classification algorithm with these information, which reduced the error of classification greatly. Some experimental results are reported.
\end{abstract}

Keywords: Classification, Pattern description, Image processing, Singular point, Flow-line, Fingerprint, Uncertainty information

\section{Introduction}

Fingerprint System is often required to handle huge data - thousands or even millions fingerprints are stored in the database. So a good classification algorithm is do needed in order to improve the system performance. In history, manual classification of fingerprints according to Henry index [1] is used in management of fingerprint database. Along with the birth of Automatic Fingerprint Identification System, the development of algorithms for automatic classification has attracted much more attentions of researchers and commercial users.

A early work for fingerprint classification was done by Kawagoe and Tojo [2]. They detected singular points and traced flow-lines around the core region as the feature, on a $32 \times 32$ directional image. Total 8 classes were used in the approach.

\footnotetext{
* Former name is Institute of Systems Science
} 
One work is presented by NIST as the classification using PCA approach [3]. Which is based on the directional image of fingerprint. After statistic learning, it is then used to classify fingerprint. Karu etc also reported a method for fingerprint classification [4]. They used the singularities of fingerprint and the direction information along the connection lines between core and deltas to decide fingerprint type. Coetzee etc presented some methods for classification by using direction image, Fourier domain comparison and direct correlation technique. However they just test 20 fingerprints. Other neural network method is also investigated to this problem [5].

All the classification research are mainly based on Henry classification scheme. In fact, there are much more information in fingerprint pattern which can be used for finer classification. In this paper, we focus on this topic. The features used here are flow-lines and singularities [6]. Structure relationship of the features are introduced as the criteria for classification of fingerprints.

\section{Image processing}

Low level image processing can enhance the perceptual quality of image with noise reduction. In AFIS system, the general stages of pre-processing include ridge orientation detection, segmentation of ridges, thinning and minutiae extraction.

\subsection{Ridge orientation detection}

Fingerprint is formed of ridges and valleys. The flow of the ridges or valleys can be represented by directional image [7], in which each entry is the local orientation of the ridge/valley and can be calculated by several methods $[4,7,3]$. In order to reduce the noise, the peak orientation in a window is used as the orientation of the center of the window.

In our experiments we found that all directional images generated from the above methods were almost same if the original fingerprint image is in good quality. If the image quality is not so good, the directional image would be incorrect. Here we use the quadrature steerable filter to estimate the ridge orientation [8]. Steerable filter is in fact a bank of filters which can steer in 2D space. So it provide a fast and powerful tool to measure the local orientation of images [9].

\subsection{Blur area and degree of fingerprint}

An important and interesting work in AFIS is how to estimate the blur degree of fingerprint image. For some fingerprint, the image in very poor quality that can not be used for automatic feature extraction. Or some zones are too blurred to be used to extract feature. For fingerprint, the knowledge we have is, the ridges directions are almost same in a small region. So the histogram of directions could 
be used as an indicator of the blur degree of a window. The direction variance in a window is another parameter for blur estimation.

Using the method of direction calculation [8], the direction energy is a natural indicator of the blur degree. A more complicated scheme to blur degree calculation can be as following:

0. To obtain the dark region (foreground) of fingerprint, i.e. fingerprint area [3].

1. To obtain directional image, for each pixel $(i, j)$, its direction is $D(i, j)$ and the strength is $S(i, j)$.

2. For each pixel $(i, j)$, to obtain local direction variance $V(i, j)$ in a window.

3. To decide the blur pixel based on $S(i, j)$ and $V(i, j)$ : for any pixel $I(i, j)$ in the image

$$
I(i, j) \text { is in blur area, if } S(i, j)<T_{s} \text { or } V(i, j)>T_{v}
$$

4. Blur degree of a fingerprint:

$$
B_{f}=\frac{\text { pixels in blurred area }}{\text { pixels in fingerprint area }}
$$

where $T_{s}$ and $T_{v}$ are predefined thresholds.

All the blurred areas are not used in the later processing. More important, it is observed that if the $B_{f}$ of a fingerprint is larger than a certain value, the fingerprint would not be processed correctly, not only in minutiae extraction but also in classification stage.

\subsection{Fingerprint skeleton extraction}

Enhancement Before skeleton extraction, fingerprint enhancement can reduce the noise greatly. In our system, the directional filtering is used for image enhancement, which can be frequency filtering, directional smoothing or steerable filtering [8] in spatial domain. After that the image, especially the short breaks of ridges and connections between ridges, can be corrected.

Segmentation Segmentation used here is to distinguish the ridges and valleys. The simplest way to perform the segmentation is a thresholding operation. However, even after ridge filtering, the image may be still noisy and varying in contrast and grey level. In order to obtain the information as correctly as possible, a local adaptive thresholding approach is developed here, with some smoothing operations $[10,11,12]$. 
Thinning and Post-processing One of the frequently used thinning methods such as those in [13] is used here. It's found that the skeleton of fingerprint is not sensitive to thinning techniques for post-processing step can eliminate the artifacts from the skeletons further. Mainly, two rules are used based on the fact that the fingerprints have mainly smooth and long ridges. One rule is used to connect the broken ridges, while the other is used to erase the short spurs, bridges and holes [12].

\section{Singularities finding and flow-lines tracing}

Singularities are those points where, in the flow field, the Poincare Index is not zero [2]. For core-like singular point, this index is $1 / 2$, that of whorl singular point is 1 and that of delta point is $-1 / 2$. In directional filed, the point with zero Poincare Index is called normal point. A special class of fingerprint is arch, where all points in the flow field are normal points, under the measurement of Poincare Index. So for arch type fingerprint, the direction variation is analyzed to obtain the arch-core point. The idea is similar to that used in [3]. After the singular points detection, further studies lead to up-core (convex) and down-core (concave).

Using the singular points captured as above, the feature line can be traced [6]. In the images without delta detected, the feature lines in the core area are the main evidence for pattern classification.

A typical result is shown in Fig.1, where the core is marked by $\bigcirc$ and delta is marked by $\triangle$. The flow lines are marked by thick lines. The direction of up-core is also marked by a cross at the up-core point. In order to deal with the noise and deformation, three flow-lines in the core region and the flow-lines traced from the delta point(s) are used for pattern analysis.

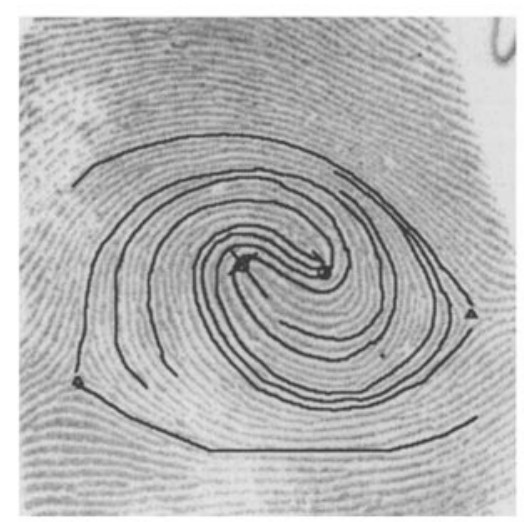

Fig. 1. Singular points and flow-lines 


\section{Classification - structural approach}

Original and manual classification of fingerprint is usually based on Henry method. Some works are also reported using this kind of classification $[14,3,4]$. One different classification method is through self-organizing mapping(SOM) neural network [5]. The classes are defined by the SOM rather than by fingerprint expert, e.g. Henry. So it is difficult to have each class with certain meaning as those in henry Classes.

Here we present a novel classification method which is based on an improved Henry Classes. The basic pattern is still obtained from the ridge flow. Considered the noise and deformation in practical system, some middle classes are introduced to treat the uncertainty.

\subsection{Improved Henry Classes}

Henry classes has five classes: ARCH, TENT, LEFT LOOP, RIGHT LOOP and WHORL. Besides, NIST of US has also introduced SCAR-class for those scarlike fingerprint. But in fact, it is often difficult to represent the scar directly. It is more like kind of noise. So we use a class "unclear type but clear fingerprint" to denote this type of fingerprint. "Clear" means the fingerprint image has good quality for there is enough contrast to distinguish the ridge and valley. "Unclear type" means that the directional image of the fingerprint is unclear, i.e. in the area, there are ridges with inconsistent directions. This kind of type can be obtained from the blur degree and type analysis. All of the classes are listed below.

\begin{tabular}{|l|l|l|}
\hline $\begin{array}{l}\text { 0. UNKNOWN (unclear } \\
\text { image) }\end{array}$ & $\begin{array}{l}\text { 1. SCAR(unclear type, } \\
\text { \& clear image }\end{array}$ & 2. ARCH \\
\hline 3. LEFT-LOOP & & \\
\hline 4. TENT/LEFT-LOOP & 5 TENT & 6. TENT/RIGHT-LOOP \\
\hline 7. RIGHT-LOOP & $\begin{array}{l}\text { 9. WHORL } \\
\text { 8. CLOCKWISE }\end{array}$ & $\begin{array}{l}\text { WHORL COUNTERCLOCKWISE } \\
\text { WHORL }\end{array}$ \\
\hline $\begin{array}{l}\text { 11. CLOCKWISE } \\
\text { WHORL with whorl }\end{array}$ & $\begin{array}{l}\text { 12. WHORL } \\
\text { with whorl }\end{array}$ & $\begin{array}{l}\text { 13. COUNTERCLOCKWISE } \\
\text { WHORL with whorl }\end{array}$ \\
\hline 14. WHORL/TWIN-LOOP & 15. LEFT-TWIN-LOOP & 16. RIGHT-TWIN-LOOP \\
\hline
\end{tabular}

Table 1. Improved Henry classes

In the classes 11,12 and 13 , there is a whorl singular point detected instead of two cores. This is different from that in [2] where a flatness parameter is 
used for defining the whorl pattern. In our scheme, the whorl/core singularity is detected from the directional image. In classification, if the distance between two detected cores is less then a threshold, the fingerprint is classified as a WHORL with whorl. Then the clockwise check is performed to obtained subclasses, 11, 12 , or 13 as in Table 1 .

\subsection{Structural Classification of Fingerprints}

From the singularities and flow-lines extracted from the fingerprint, it is possible to classify the patterns. The geometric relation of singularities provides the primary classification clue [4]. For tent/loop confused pattern, the symmetry of the trends of both sides of flow-lines and the angle $\alpha$ shown in Fig. 2 can be used to obtained the class $3,4,5,6,7$. See Fig.2.
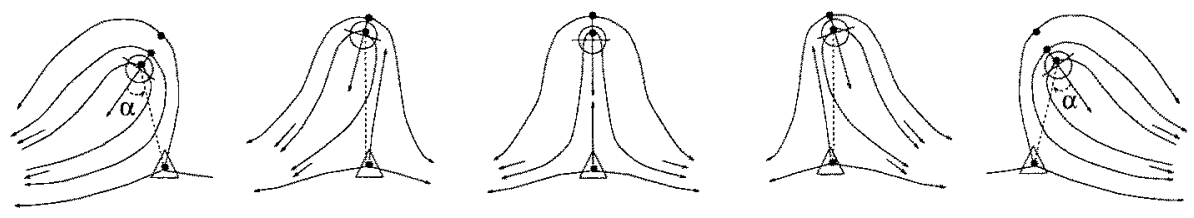

Fig. 2. Illustration of class 3 to 7

For fingerprint with a whorl singular point, it is obviously a Whorl with whorl type. Otherwise, fingerprint with two cores is whorl type. Further more, the flow-lines in core area provides the clue to decide whether the fingerprint is clockwise or counterclockwise, then classes 8-13 can be obtained. In Fig.3, from left to right and top to bottom the illustration of class 8 to 13 are shown.

The parameters are defined as $\Delta y=y(T)-y(S)$ and $\Delta x=x(P)-x(Q)$, where $S$ is the starting point of a flow-line, $P$ is the point where the flow-line has the same direction with the core direction, $Q$ is the point where the flow-line has the inverse direction with $P$ and $T$ is the point that has the same $\mathrm{x}$-value of $S$ in the Cartesian coordinates, i.e. $\mathrm{x}(\mathrm{T})=\mathrm{x}(\mathrm{S})$. So when $\Delta y>0$ and $\Delta x>0$, it is a counterclockwise whorl, see the top-left figure in Fig.3.

In practical system, due to noise, we set $\Delta y<T_{w}$ instead of $\Delta y=0$ to denote a whorl unclear of clockwise-ness. For 500dpi image, $T_{w}=5$ pixel.

For whorl/twin-loop like fingerprint, the geometric relationship between upcore and down-core is the parameter used to decide whether it is a whorl, twinloop or confused(class 14). The parameter $\alpha$ controls the result. If $|\alpha|<T_{t}$, it is a whorl type. If $\alpha \geq T_{t}$, it is LEFT-TWIN-LOOP. If $\alpha \leq-T_{t}$, it is RIGHTTWIN-LOOP. In experiments, $T_{t}$ is set to 12 degree. Further the clockwise check of the flow-lines using $\Delta y_{1}$ and $\Delta y_{2}$, combined with $\alpha$ can indicate class 15 and 16. See Fig.4. Usually, clockwise-ness of the flow-lines will be consistent with the value of $\alpha$. However they may be conflict when a fingerprint pattern is between 

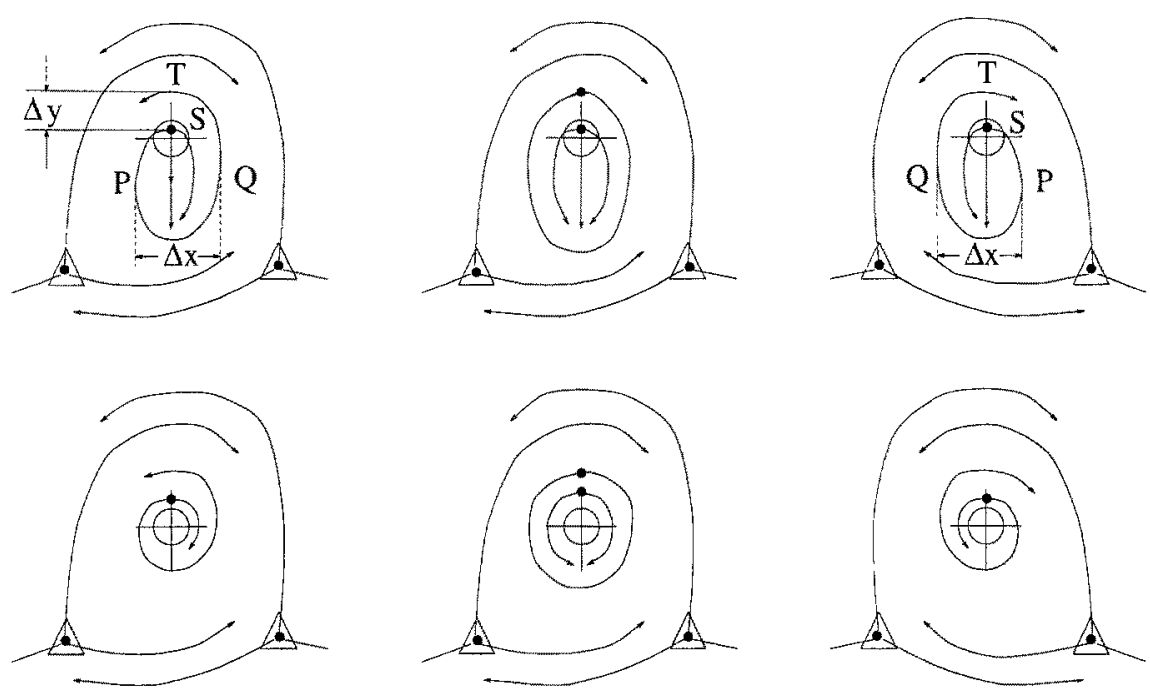

Fig. 3. Illustration of class 8 to 13

WHORL and TWIN-LOOP, deformation is too large, or noise is too strong. In this case the image is assigned as class 14 (confused).
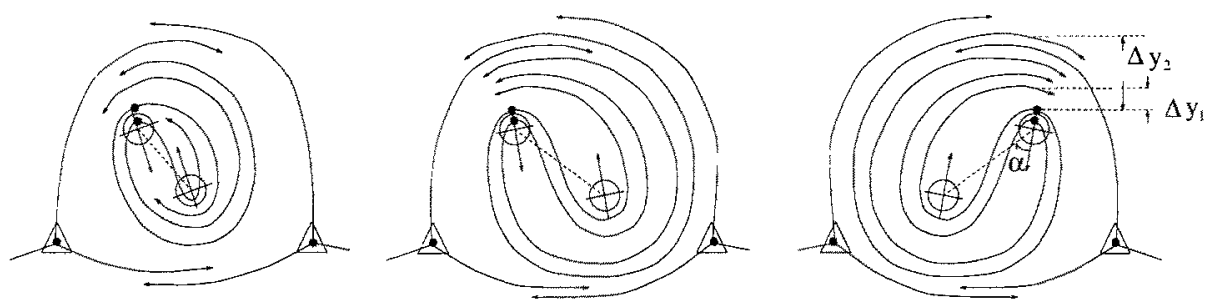

Fig. 4. Illustration of class 14 to 16

In order to indicate the fingerprints which can not be classified to any of the classes list previously, using the current information such as the singularities and flow-lines, an UNKNOWN class is defined for those images. That is the class 0 . This class is mainly composed of the images with poor quality.

\section{Experiments and discussion}

We test the approach proposed in the previous section on the database with 2700 fingerprints [3]. However, due to the database is annotated with only Henry classes, only the first four hundred fingerprints are tested. Each fingerprint is 
assigned manually with a label that indicates an improved class, see section above.

The errors occurred mainly on the fingerprints with poor quality, measured via blur degree larger than 0.60 . It causes the error in detection of singularities and tracing of flow-lines. For fingerprint in good quality, errors occurred in the classification are caused by lack of information, noise (smudge or scar) of the image caused the wrong flow-lines, and some confused cases that tent type fingerprint is classified as loop type. In table 2 the error rates of the classification are given. The first column is the top blur degree of images used in classification experiments. The second column is the best result of the classification, shown as the image number and percentage of wrong classification. Compared to the third column, the worst result, in best results all the compatible classes are taken as right classification, such as the case that a right-twin-loop type is assigned a label of whorl. But if a clockwise whorl is assigned as a right-twin-loop, it is taken as a wrong classification. It must be noted that in these experiments, the unclear type is taken as compatible with any other class. The third column is the worst result of the classification in which a right classification is defined as that any label assigned to a fingerprint is the same with that it should be.

\begin{tabular}{|c|c|c|c|}
\hline blur degree & best result(\%) & worst result(\%) & total images tested \\
\hline \hline$<0.20$ & $1(4.34)$ & $2(8.70)$ & 23 \\
\hline$<0.30$ & $2(2.82)$ & $3(4.23)$ & 71 \\
\hline$<0.40$ & $3(2.34)$ & $6(4.69)$ & 128 \\
\hline$<0.45$ & $4(2.58)$ & $9(5.81)$ & 155 \\
\hline$<0.50$ & $4(2.16)$ & $12(6.49)$ & 185 \\
\hline$<0.55$ & $4(1.81)$ & $12(5.43)$ & 221 \\
\hline$<0.60$ & $4(1.57)$ & $12(4.72)$ & 254 \\
\hline$<0.70$ & $7(2.25)$ & $19(6.11)$ & 311 \\
\hline$<0.80$ & $8(2.30)$ & $25(7.18)$ & 348 \\
\hline$<0.90$ & $15(3.91)$ & $35(9.11)$ & 384 \\
\hline$\leq 1.00$ & $17(4.25)$ & $41(10.25)$ & 400 \\
\hline
\end{tabular}

Table 2. Classification results

In the worst case, because the quality of some images are too poor which leads to UNKNOWN class, the error rate is much higher than that of best case. It is $10.25 \%$ when all the images are used in the experiments. The number of images assigned with label UNKNOWN in 41 images (see the last row of table 2) is 18 , i.e. $4.5 \%$. In the best case, the error is $4.25 \%$ when 400 images are used. This number will decrease if the blur degree decreases. Compared with the result of [4], where the error percentage is larger than $6 \%$ when the reject 
percentage is $10 \%$ in 4 -class classification, the error percentage of the proposed scheme is $5.75 \%$ in the worst case when the reject percentage is $4.5 \%$ in 16-class classification. (However they use 4000 images for testing and here we just use 400 images for testing.)

In the other wrong classification cases (not the UNKNOWN case), there are 3 kind of errors. One is that a loop type is classified as a tent or a tent is classified as loop. In fact, it is difficult to distinguish them even manually. A solution is to relax the condition of LOOP or TENT classes, see classes 4 and 6 in table 1 and Fig2. The second error is caused by lack of information. Because we did not use the flow-lines traced from concave core area, the twin-loop type and whorl type may be mis-classified. It can be solved by adding this kind of flowline information. All the other errors are caused by noise. They are extremely difficult to be corrected. This situation can only be reduced by the image quality control, in image input and preprocessing process.

\section{Summary and Conclusion}

An improved classification scheme is presented in this paper, which uses the singularities and flow-lines structure to compose the fingerprint pattern. It is shown in the experiments that the error rate of proposed classification scheme is rather low. The scheme is summarized as follows:

(1) Image ridge orientation detection. The quadrature steerable filter is used for orientation estimation.

(2) Skeleton extraction. It is found that the flow-lines tracing is some uncorrected if only based on the directional image [6]. Thus the skeletons of ridges are used here to capture the accurate flow-lines.

(3) Singularities finding and flow-lines tracing. Poincare Index is used for Singularities detection. For arch-like fingerprint, the NIST approach [3] is used for core detection. The flow-lines are traced on the directional image and skeletons of ridges.

(4) Structural classification based on the information obtained in step (3). The structure of the fingerprint pattern are used for classification.

It is observed from the experiments that the structure information can be further investigated to obtain a finer classification result. For example, the distance between a core and a delta could be used for classification. Same as the way proposed in this paper, overlapped or fuzzy classification method can work well.

The other parameters for finer classification are the flatness of the up-core, twinness of two detected cores [2], and relative core direction (relative to the link of delta and core) etc. The geometric relationships among the flow-lines traced from delta points are also the efficient feature for finer classification and are easy to be used. For large database, the proposed scheme can be a useful tool for fingerprint retrieval - the shape of the flow-lines could be used. 


\section{References}

1. E. R. Henry. Classification and Uses of Finger Prints. London: Rouledge, 1900.

2. M. Kawagoe and A. Tojo. Fingerprint pattern classification. Pattern Recognition, $17: 295-303,1984$.

3. G. T. Candela et. al. PCASYS - A Pattern-Level Classification Automation System for Fingerprints. Technical Report NISTIR 5647, Computer Systems Laboratory, Advanced Systems Division, National Institute of Standards and Technology, U.S. Dept of Commerce, Aug. 1995.

4. K. Karu and A. K. Jian. Fingerprint Classification. Pattern Recognition, 29(3):389-404, 1996.

5. U. Halici and G. Ongun. Fingerprint classification through self-organizing feature maps modified to treat uncertainties. Proceedings of the IEEE, 84(10):1497-512, 1996.

6. Weimin Huang, Gang Rong and Zhaoqi Bian. A Method of the Description of Image Information for the Classification of Fingerprints. Journal of Tsinghua University, 34(S4):107-115, 1994.

7. B. M. Mehtre, N. N. Murthy, S. Kapoor, and B. Chatterjee. Segmentation of fingerprint images using the directional image. Pattern Recognition, 20:429-435, 1987.

8. Weimin Huang, Jian-Kang Wu and Chian-Prong Lam. Fingerprint Image Enhancement with Steerable Filters. Technical Report ISS97-06, Institute of Systems Science, National University of Singapore, July. 1997.

9. William T. Freeman and Edward H. Adelson. The design and use of steerable filters. IEEE Trans on Patt. Anal. Mach. Intell, 13(9):891-906, 1991.

10. R. M. Haralick et. al. Image Segmentation Techniques. Comput. Vision Graphics Image Process., 29:100-132, 1985.

11. Louis Coetzee and Elizabeth C. Botha. Fingerprint Recogxition in Low Quality Images. Pattern Recognition, 26(10):1441-1460, 1993.

12. D. C. Douglas Hung. Enhancement and Feature Purification of Fingerprint Images. Pattern Recognition, 26(11):1661-1671, 1993.

13. S.W. Lee L. Lam and C.Y. Suen. Thinning Methodologies - A Comprehensive Survey. IEEE Trans. on Pattern Analysis and Machine Intelligence, 14:869-885, 1992.

14. C. V. K. Rao and K. Balck. Type classification of fingerprints: A syntactic approach. IEEE Trans. PAMI, 2:223-231, 1980. 